\title{
Experimental Demonstration of Mid-Infrared Computational Spectroscopy with a Plasmonic Filter Array
}

\author{
Benjamin Craig ${ }^{1}$, Vivek Raj Shrestha ${ }^{1}$, Jiajun Meng $^{2}$, and Kenneth B. Crozier ${ }^{1,2}$ \\ ${ }^{I}$ School of Physics, University of Melbourne, Victoria 3010, Australia \\ ${ }^{2}$ Department of Electrical and Electronic Engineering, University of Melbourne, Victoria 3010, Australia \\ Corresponding Author e-mail address: kenneth.crozier@unimelb.edu.au
}

\begin{abstract}
We demonstrate mid-infrared plasmonic filters. We experimentally determine the spectrum of a mid-infrared light source using an algorithm whose inputs are the total power transmitted by each filter and the transmission spectrum of each filter. (C) 2018 The Author(s)

OCIS codes: (250.5403) Plasmonics; (300.6340) Spectroscopy, infrared; (080.1753) Computation methods
\end{abstract}

\section{Introduction}

Infrared (IR) spectroscopy is important in many areas of science and industry, with the current workhorse tool being the Fourier transform infrared (FTIR) spectrometer. IR spectrometers that are smaller, lighter and less expensive than FTIR systems would be beneficial for many applications. Miniaturised spectrometer systems have recently been demonstrated that comprise an array of filters integrated with a detector array. The spectrum of the light impinging upon the array can be computationally reconstructed from the detector signals, provided the transmission spectra of the filters are known. Visible-wavelength demonstrations have been performed using filter arrays comprised of quantum dots [1] and Fabry-Perot etalons [2]. In this work, we demonstrate the fabrication of an array of 116 plasmonic filters with spectral features that span the wavelength range $1.5-19 \mu \mathrm{m}$. We use the filters to accurately determine the spectrum of a mid-infrared light source. This is done by measuring the total power transmitted by each filter, and providing the resultant column vector (116 rows) to a recursive least squares (RLS, [3]) algorithm along with the transmission spectrum of each filter. The integration of our filters with an IR detector array (eg. microbolometers) could form the basis for lightweight, compact, and inexpensive IR spectrometers.

\section{Filter Design and Fabrication}

Each filter comprises a square array of Au squares (30 nm thick, with Pd for adhesion), with an overall extent of $\sim 100 \times 100 \mu \mathrm{m}^{2}$. The substrate is an undoped $\mathrm{Si}$ wafer that is double side polished. The filters are fabricated using e-beam lithography, e-beam evaporation and lift-off. The unit cell of the filter is illustrated schematically as Fig. 1a. The period $P$ varies from 0.25 to $6 \mu \mathrm{m}$ (increment of $0.05 \mu \mathrm{m}$ ). An optical microscope image of the filter with $P=$ $6 \mu \mathrm{m}$ is shown as Fig. 1b. An optical microscope image of the 116 filters in the array is shown as Fig. 1c.
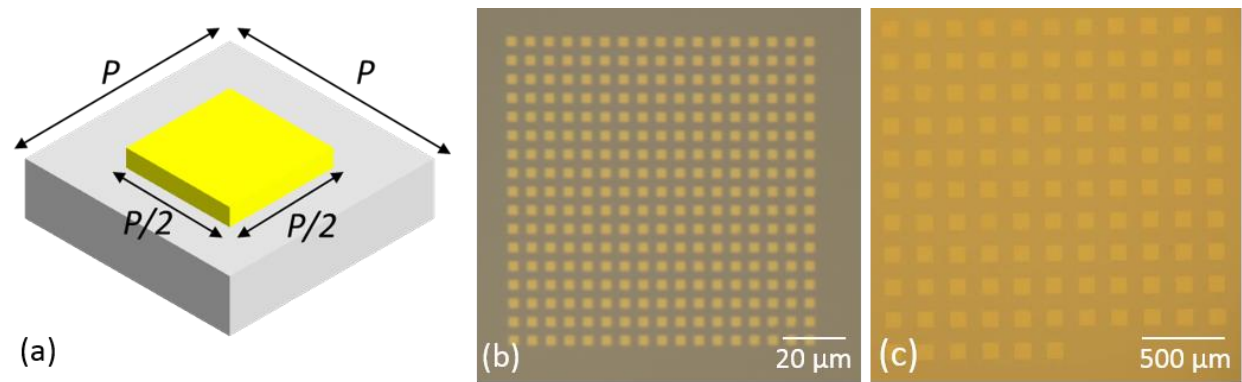

Fig. 1. (a) Unit cell of filter (period P). Optical microscope images of (b). filter with period of $6 \mu \mathrm{m}$ and (c). all 116 filters in array.

\section{Results}

Transmission spectra of the filters are measured with an FTIR microscope. The results are plotted in Fig. 2, and represent the spectra measured through each filter divided by the spectra measured through air (no sample). The measured and simulated (FDTD method) transmission spectra show agreement in the transmission dip position (Fig. 2a). There is discrepancy about the value of the transmission at resonance, with the measurements being greater than simulations. This may be because the simulations are performed at normal incidence, while the Cassegrain objective of the FTIR microscope inherently provides angled illumination. There may also be contributions from fabrication 
imperfections. All filters exhibit a transmission dip at $\lambda \sim 16 \mu \mathrm{m}$. We attribute this to absorption in the Si substrate, as it is known that Si has a large extinction coefficient around this wavelength [4].
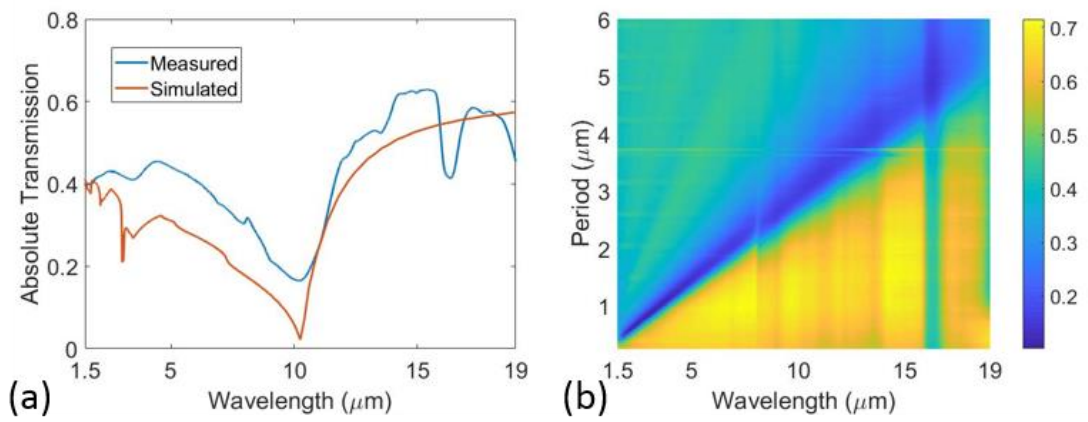

Fig. 2. (a) Simulated and measured transmission spectra of filter with $3 \mu \mathrm{m}$ period. (b) Measured transmission spectra for all 116 filters.

We next demonstrate spectroscopy using our filter array. Ideally, we would combine it with a detector array to determine the spectrum of light incident on the filter array. Each detector would measure the total optical power transmitted through each filter. However, as an appropriate detector array was not available to us, we instead perform a proof-of-principle demonstration using the FTIR microscope to measure the total optical power transmitted through each filter. We reconstruct the spectrum of the globar source of our FTIR system as follows. Transmission spectra $T(\lambda)$ of the 116 filters are measured using the FTIR microscope (Fig. 2b). We also measure the spectrum $S(\lambda)$ of light transmitted through each filter with the FTIR microscope. This represents $S(\lambda)=g(\lambda) \times M C T \_r e s p(\lambda) \times T(\lambda)$, where $g(\lambda)$ is globar power density spectrum and $M C T \_r e s p(\lambda)$ is mercury cadmium telluride (MCT) detector responsivity. For each of the 116 filters, we integrate $S(\lambda)$ over $\lambda=1.5 \mu \mathrm{m}$ to $19 \mu \mathrm{m}$. This yields a column vector $(116 \times 1)$ that is input along with $T(\lambda)$, to our RLS algorithm to estimate $g(\lambda) \times M C T_{-}$resp $(\lambda)$. The results are shown in Fig. 3a, along with the globar spectrum directly measured with the FTIR microscope. These are in very good agreement. The accuracy of the reconstruction in comparison to the spectrum measured by the FTIR is quantified by the mean-average-percentage-error (MAPE, Fig. 3b), which is defined as $M A P E=\frac{100}{N} \sum_{i=1}^{i=N}\left|\frac{T_{i}-R_{i}}{T_{i}}\right| . T_{i}$ is the spectrum ( $N=4421$ data points) directly measured by the FTIR microscope. $R_{i}$ is the reconstructed spectrum. The MAPE decreases with the number of iterations, asymptoting to $2.48 \%$. Future work will involve integration with detector arrays to yield very compact IR spectrometers.
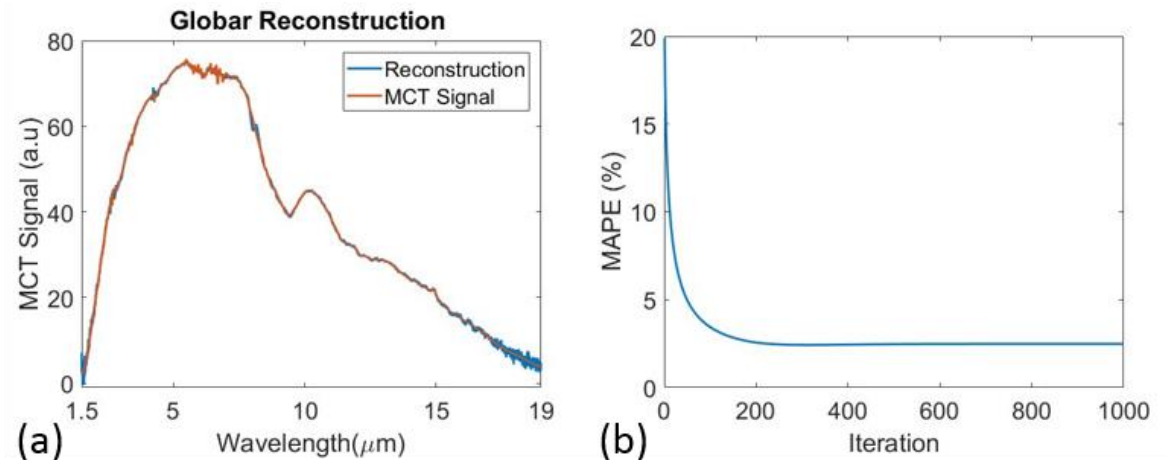

Fig. 3. (a) Reconstruction of MCT signal of globar source over a wavelength range of $1.5 \mu \mathrm{m}$ to $19 \mu \mathrm{m}, 4421$ data points were reconstructed. (b) MAPE of reconstruction versus iteration number.

\section{References}

[1] J. Bao and M. G. Bawendi, "A colloidal quantum dot spectrometer," Nature, vol. 523, pp. 67-70, 2015.

[2] E. Huang, Q. Ma and Z. Liu, "Spectrum Engineering for Reconstructive Spectrometry," in Conference on Lasers and Electro-Optics,

San Jose, CA, 2016, pp. 1-2.

[3] M. H. Hayes, "9.4: Recursive Least Squares," in Statistical Digital Signal Processing and Modeling, Wiley, 1996, p. 541.

[4] D. Chandler-Horowitz and P. M. Amirtharaj. "High-accuracy, midinfrared $\left(450 \mathrm{~cm}^{-1} \leq \omega \leq 4000 \mathrm{~cm}^{-1}\right)$ refractive index values of silicon", J. Appl. Phys. 97, 123526 (2005)

Performed in part at Melbourne Centre for Nanofabrication (MCN) in Victorian Node of Australian National Fabrication Facility (ANFF). 


\section{University Library}

\section{- M M N E R VA A gateway to Melbourne's research publications}

Minerva Access is the Institutional Repository of The University of Melbourne

Author/s:

Craig, B;Shrestha, VR;Meng, J;Crozier, KB

Title:

Experimental Demonstration of Mid-Infrared Computational Spectroscopy with a Plasmonic Filter Array

Date:

2018-01-01

Citation:

Craig, B., Shrestha, V. R., Meng, J. \& Crozier, K. B. (2018). Experimental Demonstration of Mid-Infrared Computational Spectroscopy with a Plasmonic Filter Array. 2018 CONFERENCE ON LASERS AND ELECTRO-OPTICS (CLEO), Part F92-CLEO_AT 2018, OSA \& IEEE. https://doi.org/10.1364/CLEO_AT.2018.AF3M.1.

Persistent Link:

http://hdl.handle.net/11343/294891 